\title{
Some results about the facial geometry of convex semi-infinite systems
}

\author{
M.D. Fajardo ${ }^{1}$ and M.A. López ${ }^{2}$
}

\begin{abstract}
We study the geometrical properties of the convex semi-infinite systems and their solution sets. Our main focus is on those systems enjoying the so-called locally Farkas-Minkowski property. The paper provides convex counterparts of some results already proven for linear systems, pointing out the main differences, and finding sufficient conditions for their fulfilment.
\end{abstract}

\section{Introduction}

The paper deals with convex inequality systems in the form

$$
\sigma=\left\{f_{t}(x) \leq 0, t \in T\right\},
$$

where $f_{t}: \mathbb{R}^{n} \rightarrow \mathbb{R} \cup\{+\infty\}$ is a lower semicontinuous (l.s.c., in brief) proper convex function, for $t \in T$, and $T$ is an arbitrary (possibly infinite) index set. The solution set of $\sigma$ is a (possibly empty) closed convex set $F$, and $\sigma$ is consistent if $F$ is non-empty.

If $f_{t}(x)=\left\langle a_{t}, x\right\rangle-b_{t}, t \in T$, with $x$ and $a_{t}$ in $\mathbb{R}^{n}, b_{t}$ in $\mathbb{R}$, and $\langle.,$.$\rangle represents$ the inner product in $\mathbb{R}^{n}$, we obtain the linear semi-infinite system

$$
\sigma=\left\{\left\langle a_{t}, x\right\rangle \leq b_{t}, t \in T\right\} .
$$

The main goal of the paper is to investigate the geometrical properties of the solution set $F$, and special attention is paid to those systems that satisfy the socalled locally Farkas-Minkowski property (LFM, in short).

In linear semi-infinite programming, different characterizations of the dimension of the feasible and optimal sets were provided in [1] . For linear semi-infinite systems, the LFM property was introduced in [2], while [3] gave account of the most relevant properties of the systems enjoying this property. Chapter 5 in $[\mathbf{3}]$ is devoted to the geometry of their solution sets and, in particular, Theorem 5.9 there provides

Key words and phrases. Semi-infinite convex inequalities systems, constraint qualifications, Slater condition, facial structure of the feasible set.

2000 Mathematics Subject Classification. 90C34, 52A41, 90C25, 52A20.

${ }^{1}$ Assistant Professor, Deparment of Statistics and Operations Research, Faculty of Sciences, University of Alicante, Spain.

${ }^{2}$ Professor, Deparment of Statistics and Operations Research, Faculty of Sciences, University of Alicante, Spain. 
formulas for the dimension of $F$, for its affinity, and for its topological relative interior. In this theorem the interior set of $F$ was characterized as the set of the Slater points of the system obtained from $\sigma$ by elimination of all the trivial inequalities.

For convex semi-infinite systems, the LFM property is introduced in $[\mathbf{3}$, Section 7.5], and its role as a constraint qualification for convex semi-infinite programming is emphasized there. In [4] the relationship between this constraint qualification and the upper semicontinuity (in the sense of Berge) of the so-called active and sup-active mappings is analyzed, as well as the fulfilment of the Valadier formula for the supremum function under some conditions involving the LFM property.

Section 2 of this paper studies the relative interior and the relative boundary of $F$ for a general consistent convex system. We establish similar inclusions to those which are valid in the linear case ([3, Chapter 5]). Section 3 analyzes the relationship between the concepts of face and set of carrier indices for a linear representation of $\sigma, \sigma_{L}$, and their convex counterparts. In Section 4, some appealing geometrical properties of the solution set $F$ of a convex LFM system are derived, and the main differences with the linear case are pointed out. Finally, in Section 5 , different conditions are given guaranteeing a complete characterization of the interior and the relative interior of $F$.

Let us introduce the necessary notation. Given a non-empty set $X$ of the Euclidean space $\mathbb{R}^{n}$, the convex (conical, affine, linear) hull of $X$ is denoted by conv $X$ (cone $X$, aff $X, \operatorname{span} X$, respectively), and $X^{\circ}$ represents the polar cone of $X$,

$$
X^{\circ}=\left\{y \in \mathbb{R}^{n} \mid\langle y, x\rangle \leq 0 \text { for all } x \in X\right\} .
$$

It is assumed that cone $(\emptyset)=\left\{0_{n}\right\}$, where $0_{n}$ is the null-vector in $\mathbb{R}^{n}$. We represent by $\operatorname{dim} X$ the dimension of aff $X$.

The largest subspace contained in the recession cone of $X$ is called the lineality space of $X$, and is denoted by lin $X$. A convex cone is pointed if its lineality space is reduced to the null-vector.

From the topological side, int $X, \operatorname{cl} X$ and $\operatorname{bd} X$ represent the interior, the closure and the boundary of $X$, respectively, whereas $\operatorname{rint} X$ and $\operatorname{rbd} X$ represent the relative interior and the relative boundary of $X$ (relatively to aff $X$ ), respectively.

If $f: \mathbb{R}^{n} \rightarrow \mathbb{R} \cup\{+\infty\}$ is a l.s.c. proper convex function, the effective domain, and the graph of $f$ are, respectively, the non-empty sets

$$
\operatorname{dom} f:=\left\{x \in \mathbb{R}^{n} \mid f(x)<+\infty\right\},
$$

and

$$
\operatorname{gph} f:=\left\{\left(\begin{array}{c}
x \\
f(x)
\end{array}\right) \in \mathbb{R}^{n+1} \mid x \in \operatorname{dom} f\right\} .
$$

If $x \in \operatorname{dom} f$, the one-sided directional derivative of $f$ at $x$ with respect to $v \in \mathbb{R}^{n}$

$$
f^{\prime}(x ; v)=\lim _{\lambda \downarrow 0} \frac{f(x+\lambda v)-f(x)}{\lambda}
$$

always exists $(+\infty$ and $-\infty$ being allowed as limits).

Finally, the conjugate function of $f$ is the l.s.c. proper convex function defined by 


$$
f^{*}(u)=\sup \{\langle u, x\rangle-f(x) \mid x \in \operatorname{dom} f\} .
$$

\section{Preliminary results}

Let $\sigma=\left\{f_{t}(x) \leq 0, t \in T\right\}$ be a consistent convex system. We associate with each index $t \in T$ the set

$$
F_{t}:=\left\{x \in F \mid f_{t}(x)=0\right\} .
$$

If $\sigma$ is linear, then $F_{t}=\left\{x \in F \mid\left\langle a_{t}, x\right\rangle=b_{t}\right\}$ is an exposed face of $F$, but in the convex setting, $F_{t}$ is not convex in general:

Example 1. Let $\sigma=\left\{f_{t}(x) \leq 0, t \in T\right\}$ be the convex system, in $\mathbb{R}$, where $T=[1,2]$ and

$$
f_{t}(x):=x^{2}-t .
$$

The solution set is $F=[-1,1]$, and

$$
F_{t}= \begin{cases}\{-1,1\}, & \text { if } t=1, \\ \emptyset, & \text { if } t \in] 1,2] .\end{cases}
$$

Obviously $F_{1}$ is not a convex set.

Nevertheless, the sets $F_{t}$ enjoy the same property that every convex subset of a convex set $C$ must verify in order to be a face of $C$ [5, p.162].

Proposition 1. Let $\sigma=\left\{f_{t}(x) \leq 0, t \in T\right\}$ be a consistent convex system. For any $t \in T$, every closed line segment in $F$ with a relative interior point in $F_{t}$ is entirely contained in $F_{t}$.

Proof. Suppose that $[x, y] \subset F$ and $z \in] x, y\left[\cap F_{t}\right.$. Consider $\left.\alpha \in\right] 0,1[$ such that $z=\alpha x+(1-\alpha) y$. Then, since $x$ and $y$ are in $F$,

$$
0=f_{t}(z) \leq \alpha f_{t}(x)+(1-\alpha) f_{t}(y) \leq 0,
$$

which entails $f_{t}(x)=f_{t}(y)=0$ and, so, $x$ and $y$ are also in $F_{t}$.

This proves that every closed line segment contained in $F$ such that its relative interior intersects $F_{t}$, also verifies that its endpoints are in $F_{t}$. Now we apply this result to the following line segments:

Consider $\lambda \in] 0,1\left[, \lambda \neq \alpha\right.$ and define $z_{\lambda}:=\lambda x+(1-\lambda) y$. If $\lambda>\alpha$, take the segment $\left[x, z_{\lambda}\right]$, and if $\lambda<\alpha$, take $\left[z_{\lambda}, y\right]$. In any case, $z_{\lambda} \in F_{t}$.

Corollary 1. Let $\sigma=\left\{f_{t}(x) \leq 0, t \in T\right\}$ be a consistent convex system. For any $t \in T$, the following implication holds:

$$
F_{t} \cap \operatorname{rint} F \neq \emptyset \Rightarrow F_{t}=F .
$$

Proof. Assume that $z \in F_{t} \cap \operatorname{rint} F$, and take an arbitrary $x \in F, x \neq z$. Because $z \in \operatorname{rint} F$, there will exist $\mu>1$ such that $x_{\mu}:=x+\mu(z-x) \in F$. Then $\left[x, x_{\mu}\right]$ is a closed line segment in $F$ with a relative interior point, $z$, in $F_{t}$. Hence, Proposition 1 yields $x \in\left[x, x_{\mu}\right] \subset F_{t}$ and $F \subset F_{t}$. Because the other inclusion is obvious, $F=F_{t}$. 
Definition 1. If $f_{t} \equiv 0$, the corresponding inequality in $\sigma$ is said to be trivial. We say that $t \in T$ is a proper index if $f_{t}$ is not a constant function.

The index $t \in T$ is a carrier index in $\sigma$ if $F=F_{t}$. The set of carrier indices is denoted by $T_{C}$.

Next we approach the relationship between $\operatorname{rint} F, \operatorname{rbd} F, T_{C}$ and the sets $F_{t}$, $t \in T$.

Proposition 2. Let $\sigma=\left\{f_{t}(x) \leq 0, t \in T\right\}$ be a consistent convex system, and assume that the following condition holds:

$$
F \cap I \neq \emptyset
$$

where

Then

$$
I:=\bigcap_{t \in T \backslash T_{C}} \operatorname{rint}\left(\operatorname{dom} f_{t}\right)
$$

$$
\operatorname{rint} F \subset\left\{x \in I \mid f_{t}(x)<0, t \in T \backslash T_{C}\right\} .
$$

Proof. For $t \in T$, we consider the closed convex set

$$
G_{t}=\left\{x \in \mathbb{R}^{n} \mid f_{t}(x) \leq 0\right\} .
$$

$G_{t}$ is non-empty because $\sigma$ is consistent and, obviously, $F=\bigcap_{t \in T} G_{t}$.

Let $t \in T \backslash T_{C}$. Then, there exists $x^{0} \in F$ such that $f_{t}\left(x^{0}\right)<0$. Applying Theorem 7.6 in [5], we have

$$
\operatorname{rint} G_{t}=\left\{x \in \operatorname{rint}\left(\operatorname{dom} f_{t}\right) \mid f_{t}(x)<0\right\},
$$

and

$$
\operatorname{rbd} G_{t}=\left\{G_{t} \backslash \operatorname{rint}\left(\operatorname{dom} f_{t}\right)\right\} \cup\left\{x \in \mathbb{R}^{n} \mid f_{t}(x)=0\right\} .
$$

If $(\operatorname{rint} F) \cap\left(\operatorname{rint} G_{t}\right)=\emptyset$, and since $F \subset G_{t}$, it will be

$$
\operatorname{rint} F \subset \operatorname{rbd} G_{t} \text {. }
$$

Assume that there exists $z \in \operatorname{rint} F$ such that $f_{t}(z)=0$; in other words, that $F_{t} \cap \operatorname{rint} F \neq \emptyset$. Then Corollary 1 yields $F_{t}=F$, and this contradicts $t \notin T_{C}$. From this consideration, together with (2.5) and (2.4), we get

$$
\operatorname{rint} F \subset G_{t} \backslash \operatorname{rint}\left(\operatorname{dom} f_{t}\right) \text {. }
$$

Since $G_{t} \backslash \operatorname{rint}\left(\operatorname{dom} f_{t}\right)$ is closed,

$$
F=\operatorname{cl} F=\operatorname{cl}(\operatorname{rint} F) \subset G_{t} \backslash \operatorname{rint}\left(\operatorname{dom} f_{t}\right),
$$

and this contradicts the assumption (2.1).

Then we have concluded that $(\operatorname{rint} F) \cap\left(\operatorname{rint} G_{t}\right) \neq \emptyset$ and, by Theorem 6.5 in $[5]$,

$$
\operatorname{rint} F=\operatorname{rint}\left(F \cap G_{t}\right)=(\operatorname{rint} F) \cap\left(\operatorname{rint} G_{t}\right),
$$

which entails

$$
\operatorname{rint} F \subset \operatorname{rint} G_{t} \text {. }
$$


When the functions $f_{t}, t \in T$, are all finite-valued, (2.1) is satisfied trivially with $I=\mathbb{R}^{n}$. On other hand, is clear that if the set $I$ is empty, the inclusion (2.3) cannot hold. Next we provide a relaxation of (2.3), extending Theorem 5.1 in [3], and that does not require any additional condition as (2.1). Then

Proposition 3. Let $\sigma=\left\{f_{t}(x) \leq 0, t \in T\right\}$ be a consistent convex system.

$$
\operatorname{rint} F \subset\left\{x \in \mathbb{R}^{n} \mid f_{t}(x)<0, t \in T \backslash T_{C} ; f_{t}(x)=0, t \in T_{C}\right\},
$$

and

$$
\operatorname{rbd} F \supset \bigcup_{t \in T \backslash T_{C}} F_{t} \text {. }
$$

Proof. Let $t \in T \backslash T_{C}$ and $G_{t}=\left\{x \in \mathbb{R}^{n} \mid f_{t}(x) \leq 0\right\}$.

If $z \in \operatorname{rint} F \subset G_{t}$ is such that $f_{t}(z)=0$, Corollary 1 leads us to $F_{t}=F$, in contradiction with $t \notin T_{C}$. Thus

$$
\operatorname{rint} F \subset\left\{x \in \mathbb{R}^{n} \mid f_{t}(x)<0\right\} .
$$

Moreover, if $t \in T_{C}$,

$$
\operatorname{rint} F \subset F \subset\left\{x \in \mathbb{R}^{n} \mid f_{t}(x)=0\right\} .
$$

Then, (2.7) follows from (2.9) and (2.10), and (2.8) is a trivial consequence of (2.7), by complementarity.

Remark 1. In the linear case, and according to Theorem 5.1 in [3], bd $F$ includes the union of all the faces $F_{t}$ of proper indices; i.e., those indices such that $a_{t} \neq 0_{n}$.

In the convex case, the following example shows that we can find a proper index $t$ such that $F_{t}$ is not contained in bd $F$.

Example 2. Let $\sigma=\left\{f_{t}(x) \leq 0, t \in T\right\}$ be the system, in $\mathbb{R}$, for which

$$
f_{t}(x):=\max \left\{0, t x^{2}-t\right\}, T=[1,2] .
$$

Observe that $F=[-1,1]=F_{t}$, for all $t \in T$, whereas bd $F=\{-1,1\}$.

Corollary 4 , in the following section, establishes a sufficient condition that, in the convex case, guarantees the inclusion $F_{t} \subset$ bd $F$, for any index $t$.

Definition 2. A solution $x^{0}$ of $\sigma=\left\{f_{t}(x) \leq 0, t \in T\right\}$ is said to be a Slater point of $\sigma$ if it satisfies all the inequalities strictly; i.e., if $f_{t}\left(x^{0}\right)<0$, for all $t \in T$.

Corollary 2. Let $\sigma=\left\{f_{t}(x) \leq 0, t \in T\right\}$ be a consistent convex system. Then $\sigma$ has a Slater point if and only if $T_{C}=\emptyset$.

Proof. Let us start by assuming the existence of a Slater point; i.e., a point $x^{0} \in F$ such that $f_{t}\left(x^{0}\right)<0$, for all $t \in T$. Then $F \neq F_{t}$, for every $t \in T$, and $T_{C}=\emptyset$.

For the converse implication, if $T_{C}=\emptyset$, Proposition 3 allows us to write

$$
\operatorname{rint} F \subset\left\{x \in \mathbb{R}^{n} \mid f_{t}(x)<0, t \in T\right\} .
$$

Since $F$ is a non-empty convex set, $\operatorname{rint} F \neq \emptyset$ and there exists a Slater point for $\sigma$. 
Definition 3. An index $t \in T$ (or the constraint $f_{t}(x) \leq 0$ ) is active at $\bar{x} \in F$ if $f_{t}(\bar{x})=0$. The set of active indices at $\bar{x} \in F$ is

$$
T(\bar{x})=\left\{t \in T \mid f_{t}(\bar{x})=0\right\},
$$

and the active cone at $\bar{x} \in F$ is

$$
A(\bar{x}):=\text { cone }\left(\bigcup\left\{\partial f_{t}(\bar{x}), t \in T(\bar{x})\right\}\right),
$$

where $\partial f_{t}(\bar{x})$ represents the subdifferential set of $f_{t}$ at $\bar{x}$.

Remember that $\operatorname{dom} f_{t}=\mathbb{R}^{n}$ implies that $\partial f_{t}(\bar{x})$ is a non-empty compact set [5, Theorem 23.4]. In the linear case, $A(\bar{x})=$ cone $\left\{a_{t}, t \in T(\bar{x})\right\}$.

Definition 4. A consistent convex system $\sigma=\left\{f_{t}(x) \leq 0, t \in T\right\}$ is tight when $\operatorname{dim} A(\bar{x})>0$, for every $\bar{x} \in \operatorname{bd} F$.

In the linear case, a tight system verifies that bd $F$ is the union of all the faces $F_{t}$ of proper indices [3, p. 103]. Again, this property might fail for a convex system, as Example 2 shows. Instead of that, we have the following result:

Proposition 4. Let $\sigma=\left\{f_{t}(x) \leq 0, t \in T\right\}$ be a tight convex system. Then bd $F$ is included in the union of all the sets $F_{t}$ associated with proper indices.

Proof. If $\bar{x} \in \mathrm{bd} F$, then $\operatorname{dim} A(\bar{x})>0$, and hence, there exist $t \in T(\bar{x})$ and $v \in \partial f_{t}(\bar{x})$ such that $v \neq 0_{n}$. This implies that $\bar{x} \in F_{t}$ and $t$ is a proper index (if $f_{t}$ were a constant function, then $\left.\partial f_{t}(\bar{x})=\left\{0_{n}\right\}\right)$.

The inclusion established in the last proposition can be strict, as Example 2 shows.

Corollary 3. Let $\sigma=\left\{f_{t}(x) \leq 0, t \in T\right\}$ be a tight convex system. If $\sigma$ has a Slater point, then bd $F$ is the union of all the sets $F_{t}, t \in T$.

Proof. By Corollary 2, $T_{C}=\emptyset$, and, by Proposition 3 , bd $F$ includes the union of all the sets $F_{t}, t \in T$ (since bd $F \supset \operatorname{rbd} F$ ). On the other hand, if $t \in T$ is not a proper index, then $f_{t}$ will be a negative constant function (if $f_{t} \equiv 0$, then $t \in T_{C}$, and it cannot be positive, because $\sigma$ is consistent), hence $F_{t}=\emptyset$. Since $\sigma$ is tight, we get the statement thanks to Proposition 4.

\section{Linear representation of a convex system}

Let us associate with a convex system $\sigma=\left\{f_{t}(x) \leq 0, t \in T\right\}$ the following system of linear inequalities:

$$
\sigma_{L}=\left\{\langle u, x\rangle \leq\langle u, y\rangle-f_{t}(y), t \in T, y \in \operatorname{dom} \partial f_{t}, u \in \partial f_{t}(y)\right\} .
$$

It is easy to verify that $\sigma_{L}$ and $\sigma$ have the same solution set, $F$, and, for this reason, it is said that $\sigma_{L}$ and $\sigma$ are equivalent. This provides a linear representation of $F$, whose indices set is

$$
T_{L}:=\left\{(t, y, u), t \in T, y \in \operatorname{dom} \partial f_{t}, u \in \partial f_{t}(y)\right\} .
$$

For each index $(t, y, u) \in T_{L}$, the associated exposed face will be denoted by $F_{(t, y, u)}$; i.e.,

$$
F_{(t, y, u)}:=\left\{x \in F \mid\langle u, x\rangle=\langle u, y\rangle-f_{t}(y)\right\} .
$$


The set of carrier indices for $\sigma_{L}$ is denoted by $T_{L, C}$.

Next we establish the relationships between the sets $F_{t}, F_{(t, y, u)}, T_{C}$ and $T_{L, C}$.

Proposition 5. Let $\sigma=\left\{f_{t}(x) \leq 0, t \in T\right\}$ be a consistent convex system. Then, the following statements hold:

(i) If $t \in T$, then $F_{(t, y, u)} \subset F_{t}$, for all $y \in \operatorname{dom} \partial f_{t}$ and every $u \in \partial f_{t}(y)$.

(ii) If $t \in T$, and $F \subset \operatorname{dom} \partial f_{t}$, then

$$
F_{t}=\bigcup\left\{F_{(t, x, u)} \mid x \in F, u \in \partial f_{t}(x)\right\} .
$$

(iii) If $F_{t} \neq \emptyset$ is an exposed face of $F$, and $F \subset \operatorname{dom} \partial f_{t}$, there will exist $\bar{x} \in F$ and $\bar{u} \in \partial f_{t}(\bar{x})$ such that $F_{t}=F_{(t, \bar{x}, \bar{u})}$.

(iv) If $(t, y, u) \in T_{L, C}$, then $t \in T_{C}$. In particular $T_{C}=\emptyset$ entails $T_{L, C}=\emptyset$.

(v) If $F \cap \operatorname{rint}\left(\operatorname{dom} f_{t}\right) \neq \emptyset$, for all $t \in T$, then $T_{L, C}=\emptyset$ implies $T_{C}=\emptyset$.

(vi) If $F \cap \operatorname{rint}\left(\operatorname{dom} f_{t}\right) \neq \emptyset$, for all $t \in T$, and $\bar{t} \in T_{C}$, then there exists $x \in F$ and $u \in \partial f_{\bar{t}}(x)$ such that $(\bar{t}, x, u) \in T_{L, C}$.

(vii) If $t \in T \backslash T_{C}$ and $F \subset \operatorname{dom} \partial f_{t}$, then

$$
F_{t}=\bigcup\left\{F_{(t, x, u)} \mid x \in \operatorname{rbd} F, u \in \partial f_{t}(x)\right\} .
$$

Proof. (i) If $F_{(t, y, u)}=\emptyset$ the inclusion is trivial. Otherwise, take $x \in F_{(t, y, u)}$. Since $x \in F$, we have $f_{t}(x) \leq 0$. Moreover,

$$
f_{t}(x) \geq f_{t}(y)+\langle u, x-y\rangle=0 .
$$

Then $f_{t}(x)=0$, which implies that $x \in F_{t}$.

(ii) Since $F \subset \operatorname{dom} \partial f_{t}$, and according to (i), $F_{(t, x, u)} \subset F_{t}$, for every $x \in F$ and all $u \in \partial f_{t}(x)$.

To show the converse inclusion, take $x \in F_{t}$. Since $x \in F$ and $f_{t}(x)=0$, we consider the index $(t, x, u)$ in $T_{L}$ where $u \in \partial f_{t}(x) \neq \emptyset$. Then $\langle u, x\rangle=\langle u, x\rangle-f_{t}(x)$, and we have $x \in F_{(t, x, u)}$.

(iii) We know from (i) that $F_{(t, x, u)} \subset F_{t}$, for all $x \in F$ and every $u \in \partial f_{t}(x)$. We want to show that there exist $\bar{x} \in F$ and $\bar{u} \in \partial f_{t}(\bar{x})$ such that $F_{t} \subset F_{(t, \bar{x}, \bar{u})}$.

If we prove that $\left(\operatorname{rint} F_{t}\right) \cap F_{(t, \bar{x}, \bar{u})} \neq \emptyset$ for some $(t, \bar{x}, \bar{u}) \in T_{L}$, Theorem 18.1 in [5] yields the inclusion we are looking for.

Hence, suppose that $\left(\operatorname{rint} F_{t}\right) \cap F_{(t, x, u)}=\emptyset$ for all $(t, x, u)$, with $x \in F$ and $u \in \partial f_{t}(x)$. Then

$$
\left(\operatorname{rint} F_{t}\right) \cap\left(\bigcup\left\{F_{(t, x, u)} \mid x \in F, u \in \partial f_{t}(x)\right\}\right)=\emptyset,
$$

and by (ii), we get ( $\left.\operatorname{rint} F_{t}\right) \cap F_{t}=\emptyset$, and this is a contradiction because $F_{t} \neq \emptyset$.

(iv) If $(t, y, u) \in T_{L, C}$ then $F_{(t, y, u)}=F$ and by (i), $F \subset F_{t}$, hence $F=F_{t}$.

(v) If $T_{L, C}=\emptyset$, according to Corollary 5.1 .1 in $[\mathbf{3}], \sigma_{L}$ has a Slater point. We shall prove that $\sigma$ also has a Slater point, and Corollary 2 applies to conclude $T_{C}=\emptyset$.

Let $x^{0} \in F$ be a Slater point of $\sigma_{L}$. Assume first that $x^{0} \in \operatorname{rbd} F$ and take $z \in \operatorname{rint} F$. Then, for all $\lambda, 0<\lambda<1$, we can easily prove that $(1-\lambda) z+\lambda x^{0} \in \operatorname{rint} F$ is also a Slater point of $\sigma_{L}$.

Hence, we consider only the possibility $x^{0} \in \operatorname{rint} F$. 
Now, Corollary 6.5.2 in [5] leads us to the inclusion $\operatorname{rint} F \subset \operatorname{rint}\left(\operatorname{dom} f_{t}\right)$, for all $t \in T$, and, therefore, $\partial f_{t}\left(x^{0}\right) \neq \emptyset$, for all $t \in T$. If $f_{t}\left(x^{0}\right)=0$ for some $t \in T$, we can take the index $\left(t, x^{0}, u\right)$ in $T_{L}$ (with $u \in \partial f_{t}\left(x^{0}\right)$ ). The associated constraint will be active at $x^{0}$, and we shall get a contradiction.

(vi) Since $\bar{t} \in T_{C}, F_{\bar{t}}=F$. First, we shall show that $F \subset \operatorname{dom} \partial f_{\bar{t}}$.

In the case $\operatorname{dim} F=0$, there is nothing to prove, because $F \cap \operatorname{rint}\left(\operatorname{dom} f_{\bar{t}}\right) \neq \emptyset$ implies $F \subset \operatorname{rint}\left(\operatorname{dom} f_{\bar{t}}\right) \subset \operatorname{dom} \partial f_{\bar{t}}$. Hence, let us suppose that $\operatorname{dim} F>0$.

We know already that $\operatorname{rint} F \subset \operatorname{rint}\left(\operatorname{dom} f_{\bar{t}}\right) \subset \operatorname{dom} \partial f_{\bar{t}}$. If $F$ were not included in dom $\partial f_{\bar{t}}$, there would exist $\bar{x} \in \operatorname{rbd} F$ such that $\partial f_{\bar{t}}(\bar{x})=\emptyset$. According to Theorem 23.3 in [5], for all $z \in \operatorname{rint}\left(\operatorname{dom} f_{\bar{t}}\right)$,

$$
f_{\bar{t}}^{\prime}(\bar{x} ; z-\bar{x})=-\infty .
$$

In particular, for all $z \in \operatorname{rint} F$,

$$
\lim _{\lambda \downarrow 0} \frac{f_{\bar{t}}(\bar{x}+\lambda(z-\bar{x}))}{\lambda}=-\infty,
$$

because $f_{\bar{t}}(\bar{x})=0$. But, for $0<\lambda \leq 1$,

$$
\bar{x}+\lambda(z-\bar{x})=(1-\lambda) \bar{x}+\lambda z \in \operatorname{rint} F,
$$

and

$$
\frac{f_{\bar{t}}(\bar{x}+\lambda(z-\bar{x}))}{\lambda}=0,
$$

contradicting (3.2). Hence, $F \subset \operatorname{dom} \partial f_{\bar{t}}$.

By (iii), we get $F_{\bar{t}}=F=F_{(\bar{t}, x, u)}$, for some $x \in F$ and some $u \in \partial f_{\bar{t}}(x)$.

(vii) For every $t \in T$, (ii) entails

$$
\bigcup\left\{F_{(t, x, u)} \mid x \in \operatorname{rbd} F, u \in \partial f_{t}(x)\right\} \subset F_{t} .
$$

For $t \in T \backslash T_{C},(2.8)$ yields $F_{t} \subset \operatorname{rbd} F$. Hence, if $\bar{x} \in F_{t}$, we have $\bar{x} \in F_{(t, \bar{x}, \bar{u})}$, for every $\bar{u} \in \partial f_{t}(\bar{x})$. Then

Corollary 4. Let $\sigma=\left\{f_{t}(x) \leq 0, t \in T\right\}$ be a consistent convex system.

$$
\operatorname{bd} F \supset \bigcup_{t \in T} F_{t},
$$

provided that one of the following conditions holds:

(i) $T_{C}=\emptyset$;

(ii) $T_{C} \neq \emptyset, F \cap \operatorname{rint}\left(\operatorname{dom} f_{t}\right) \neq \emptyset$, for all $t \in T$, and $\inf f_{t}<0$ for each $t \in T_{C}$.

Proof. (i) If $T_{C}=\emptyset$, then the statement is true according to (2.8).

(ii) Take $t \in T \backslash T_{C}$. Then, again by (2.8), $F_{t} \subset \operatorname{rbd} F \subset$ bd $F$.

Take now, $t \in T_{C}$. We shall show that bd $F=F=F_{t}$.

Since $t \in T_{C}$, by Proposition 5 (vi), there exist $x \in F$ and $u \in \partial f_{t}(x)$ such that $(t, x, u) \in T_{L, C}$. Then

$$
F \subset\left\{y \in \mathbb{R}^{n} \mid\langle u, y\rangle=\langle u, x\rangle-f_{t}(x)\right\} .
$$

But $f_{t}(x)=0$, and $0_{n} \notin \partial f_{t}(x)$ (because inf $\left.f_{t}<0\right)$. Then we have 


$$
F \subset\left\{y \in \mathbb{R}^{n} \mid\langle u, y\rangle=\langle u, x\rangle\right\},
$$

with $u \neq 0_{n}$. This implies int $F=\emptyset$ and, hence, bd $F=F$.

Remark 2. The condition $F \subset \operatorname{dom} \partial f_{t}$ cannot be suppressed in Proposition 5 (ii) and (iii), as the following examples show.

ExAmple 3 . Let us consider a convex system, in $\mathbb{R}$, with a unique inequality, $\sigma=\left\{f_{0}(x) \leq 0\right\}$, where

$$
f_{0}(x):= \begin{cases}-\left(1-x^{2}\right)^{\frac{1}{2}}, & \text { if }|x| \leq 1 \\ +\infty & \text { if }|x|>1\end{cases}
$$

Observe that $F=[-1,1]$. The function $f_{0}$ is subdifferentiable (in fact, differentiable) at $x$, when $|x|<1$, but $\partial f_{0}(x)=\emptyset$ if $|x|=1$. We get $F_{0}=\{-1,1\}$ and, for all $\left.x \in F \cap \operatorname{dom} \partial f_{0}=\right]-1,1[$,

$$
\partial f_{0}(x)=\left\{\nabla f_{0}(x)\right\}=\left\{x\left(1-x^{2}\right)^{-\frac{1}{2}}\right\} .
$$

Then, for all $x \in]-1,1[$, we have

$$
F_{\left(0, x, \nabla f_{0}(x)\right)}=\{y \in F \mid y x=1\}=\emptyset
$$

and, consequently,

$$
\bigcup\left\{F_{(0, x, u)} \mid x \in F \cap \operatorname{dom} \partial f_{0}, u \in \partial f_{0}(x)\right\}=\emptyset \neq F_{1} .
$$

Example 4 . Let $\sigma=\left\{f_{0}(x) \leq 0\right\}$ be a convex system, in $\mathbb{R}$, with

$$
f_{0}(x):= \begin{cases}-x^{\frac{1}{2}}, & \text { if } x \geq 0 \\ +\infty & \text { if } x<0\end{cases}
$$

We have $F=\left[0,+\infty\left[\right.\right.$. The function $f_{0}$ is differentiable at $x>0$, with $\nabla f_{0}(x)=$ $-(1 / 2) x^{-(1 / 2)}$, but $\partial f_{0}(0)=\emptyset$. Moreover, $F_{0}=\{0\}$, is an exposed face of $F$, and for all $x>0$,

$$
F_{\left(0, x, \nabla f_{0}(x)\right)}=\{y \in F \mid y=-x\}=\emptyset .
$$

Remark 3. Finally, in Proposition 5, if $F \subset \operatorname{rbd}\left(\operatorname{dom} f_{t}\right)$, for some $t \in T$, (v) can fail, as we can see in the following example:

Example 5. Let $\sigma=\left\{f_{t}(x) \leq 0, t \in T\right\}$ be the convex system, in $\mathbb{R}$, where $T=\{0\} \cup \mathbb{N}$, and

$$
\begin{aligned}
& f_{0}(x):= \begin{cases}-\left(1-x^{2}\right)^{\frac{1}{2}}, & \text { if }|x| \leq 1 \\
+\infty & \text { if }|x|>1\end{cases} \\
& f_{r}(x):=|x-1|-\frac{1}{r}, r \in \mathbb{N}
\end{aligned}
$$

We have $F=\{1\} \subset \operatorname{rbd}\left(\operatorname{dom} f_{0}\right)$, and $T_{C}=\{0\}$.

In this case $\sigma_{L}$ is the system composed by the following inequalities (without repetition): 


$$
\left\{t x \leq 1,|t|<1, x \leq 1+\frac{1}{s}, s>0,-x \leq-1+\frac{1}{u}, u>0\right\}
$$

and we conclude $T_{L, C}=\emptyset$.

This example also shows that (vi) could fail if $F \cap \operatorname{rint}\left(\operatorname{dom} f_{\bar{t}}\right)=\emptyset$, for some $\bar{t} \in T$ (here $\bar{t}=0$ ).

\section{Locally Farkas-Minkowski systems}

Definition 5. A consistent convex system $\sigma$ is locally Farkas-Minkowski (LFM) if

$$
D(F, \bar{x})^{\circ}=A(\bar{x}), \text { for all } \bar{x} \in F,
$$

where $D(F, \bar{x})$ is the cone of feasible directions to $F$ at $\bar{x}$; i.e.

$$
D(F, \bar{x}):=\left\{y \in \mathbb{R}^{n} \mid \bar{x}+\mu y \in F, \text { for some } \mu>0\right\},
$$

and $A(\bar{x})$ is the active cone at $\bar{x}$ introduced in Definition 3.

Observe that $D(F, \bar{x})^{\circ}$ is nothing else that the normal cone to $F$ at $\bar{x}$, also represented by $N(F, \bar{x})$. As a consequence of Lemma 2.1 in [4], the LFM property should be investigated only at the boundary feasible points (if $\sigma$ is LFM, one has $A(\bar{x}) \neq\left\{0_{n}\right\}$ for every $\bar{x} \in \operatorname{bd}(F)$; i.e., $\sigma$ is tight). Also, it is shown in Theorem 7.10 in $[\mathbf{3}]$ that $\sigma$ is LFM if and only if $\sigma_{L}$ is LFM.

This property plays an important role as a constraint qualification in ordinary non-linear programming, where it is called Basic Constraint Qualification (BCQ, in brief). See, for instance [6, pp. 307-309]. In convex semi-infinite programming, its role as constraint qualification has been proved in [3, Theorem 7.8], and its relationship with the Slater condition appears in [3, Theorem 7.9]. Li, Nahak and Singer, in [7], give characterizations of the LFM property (using the term BCQ) through the closedness of certain associated convex cone-valued mappings. Also, assuming the BCQ, formulas for the distance of a point to the solution set of a semi-infinite system of convex inequalities are given.

Our principal objective is to provide a deeper knowledge of the geometrical behaviour of the LFM convex systems. For the sake of simplicity, in this section we shall consider only finite-valued convex functions; i.e., $\operatorname{dom} f_{t}=\mathbb{R}^{n}$, for all $t \in T$ (see, for instance, $[\mathbf{7}]$ ).

The next Theorem provides convex counterparts of the results established in [3, Theorem 5.9].

THEOREM 1. The following statements are valid for any LFM convex system $\sigma=\left\{f_{t}(x) \leq 0, t \in T\right\}:$

(i) For every $\bar{x} \in F$

$$
\operatorname{lin} A(\bar{x})=(F-\bar{x})^{\perp}=\operatorname{span}\left\{\cup_{t \in T_{C}} \cap_{y \in F} \partial f_{t}(y)\right\} .
$$

(ii) If $T_{C} \neq \emptyset$, then

$$
\text { aff } F=\left\{x \in \mathbb{R}^{n} \mid\langle u, x\rangle=\langle u, y\rangle, u \in \cup_{t \in T_{C}} \cap_{y \in F} \partial f_{t}(y)\right\} .
$$

(iii) If $T_{C}=\emptyset$, then int $F$ is the set of Slater points of $\sigma$. 
(iv) If $\langle a, x\rangle \leq b$ is a supporting half-space to $F$ defining an exposed face $E$, then $a \in A(y)$ for all $y \in E$, and $E$ is contained in the intersection of a finite number of sets $F_{t}$ associated with proper indices.

Proof. (i) Given $\bar{x} \in F$, and denoting by $A_{L}(\bar{x})$ the active cone at $\bar{x}$ corresponding to $\sigma_{L}$, we have $A(\bar{x})=A_{L}(\bar{x})$, according to the proof of Theorem $7.10 \mathrm{in}$ [3]. Then, Theorem 5.9 (i) in [3], yields

$$
\operatorname{lin} A(\bar{x})=(F-\bar{x})^{\perp}=\operatorname{span}\left\{u \in \mathbb{R}^{n} \mid(t, y, u) \in T_{L, C}\right\} .
$$

We use the notation

$$
X:=\left\{u \in \mathbb{R}^{n} \mid(t, y, u) \in T_{L, C}\right\},
$$

and

$$
Y:=\cup_{t \in T_{C}} \cap_{y \in F} \partial f_{t}(y) .
$$

First, we shall see that $X \subset Y$.

If $u \in X$, there exist $y \in \mathbb{R}^{n}$ and $t \in T$ such that $u \in \partial f_{t}(y)$ and $(t, y, u) \in T_{L, C}$. By Proposition 5 (iv), $t \in T_{C}$. Now we have to prove that $u \in \partial f_{t}(x)$ for all $x \in F$. Since $F_{(t, y, u)}=F, f_{t}(y)=\langle u, y-x\rangle$, for all $x \in F$.

On the other hand, for all $z \in \mathbb{R}^{n}, f_{t}(z) \geq f_{t}(y)+\langle u, z-y\rangle$. Replacing $f_{t}(y)$ by $\langle u, y-x\rangle$, we obtain

$$
f_{t}(z) \geq\langle u, y-x\rangle+\langle u, z-y\rangle=\langle u, z-x\rangle,
$$

and, since $f_{t}(x)=0$, we can write, for all $z \in \mathbb{R}^{n}$,

$$
f_{t}(z) \geq f_{t}(x)+\langle u, z-x\rangle,
$$

for all $x \in F$, and we get $u \in \partial f_{t}(x)$ for all $x \in F$. Thus $u \in Y$ and we have $\operatorname{span} X \subset \operatorname{span} Y$.

To see the opposite inclusion, we prove that $\operatorname{span} Y \subset(F-\bar{x})^{\perp}=\operatorname{span} X$, according to (4.3).

Take $v \in Y$. Then there exists $t \in T_{C}$ such that $v \in \partial f_{t}(y)$, for all $y \in F$. Since $v \in \partial f_{t}(\bar{x}), f_{t}(y) \geq f_{t}(\bar{x})+\langle v, y-\bar{x}\rangle$ for all $y \in F$, and similarly, $f_{t}(\bar{x}) \geq$ $f_{t}(y)+\langle v, \bar{x}-y\rangle$ for all $y \in F$. Taking into account that $f_{t}(x)=0$, for all $x \in F$, we get $v \in(F-\bar{x})^{\perp}$.

(ii) First, observe that for $u \in Y$ and according to (i), $\langle u, y\rangle$ is constant, for all $y \in F$. Consequently, the equalities in (4.2) are well defined.

If $T_{C} \neq \emptyset$, then $T_{L, C} \neq \emptyset$, and by Theorem 5.9 (iii) in [3] we have

$$
\text { aff } F=\left\{x \in \mathbb{R}^{n} \mid\langle u, x\rangle=\langle u, y\rangle-f_{t}(y),(t, y, u) \in T_{L, C}\right\} .
$$

Denoting

$$
Z:=\left\{x \in \mathbb{R}^{n} \mid\langle u, x\rangle=\langle u, y\rangle, u \in \cup_{t \in T_{C}} \cap_{y \in F} \partial f_{t}(y)\right\},
$$

we shall see first that aff $F \subseteq Z$.

Take $x \in$ aff $F$. Then, by (4.4), $\langle u, x\rangle=\langle u, y\rangle-f_{t}(y)$ for all $(t, y, u) \in T_{L, C}$. If $t \in T_{C}$ and $u \in \cap_{y \in F} \partial f_{t}(y)$, then $(t, z, u) \in T_{L, C}$, for all $z \in F$ (otherwise, there would exist $z \in F$ such that $(t, z, u) \in T_{L} \backslash T_{L, C}$, and this implies the existence of $z^{0} \in F$ verifying $\left\langle u, z^{0}\right\rangle\left\langle\langle u, z\rangle-f_{t}(z)\right.$. But $f_{t}(z)=0$, hence $\left\langle u, z^{0}\right\rangle<\langle u, z\rangle$, 
contradicting the fact that $\langle u, w\rangle$ is constant for all $w \in F)$. We conclude that $\langle u, x\rangle=\langle u, z\rangle$, for all $z \in F$ and $x \in Z$.

Now, take $x \in Z$, and $(t, y, u) \in T_{L, C}$. It has been shown in (i) that $t \in T_{C}$ and $u \in \partial f_{t}(z)$ for all $z \in F$; hence $\langle u, x\rangle=\langle u, z\rangle$, for all $z \in F$. On the other hand, $F_{(t, y, u)}=F$, and for all $z \in F$, we have $\langle u, z\rangle=\langle u, y\rangle-f_{t}(y)$. We conclude that $\langle u, x\rangle=\langle u, y\rangle-f_{t}(y)$, and therefore $x \in \operatorname{aff} F$.

(iii) If $T_{C}=\emptyset$, then $T_{L, C}=\emptyset$, according to Proposition $5(\mathrm{v})$. Thus there are no trivial inequalities in $\sigma_{L}$, and according to Theorem 5.9 (iv) in [3], int $F$ can be expressed in the form

$$
\left\{x \in \mathbb{R}^{n} \mid\langle u, x\rangle<\langle u, y\rangle-f_{t}(y),(t, y, u) \in T_{L}\right\} .
$$

We shall prove that this is the set of the Slater points of $\sigma$.

First, take $x \in F$ such that $f_{t}(x)<0$, for every $t \in T$. Now, if $(t, y, u) \in T_{L}$, then $0>f_{t}(x) \geq f_{t}(y)+\langle u, x-y\rangle$; hence $\langle u, x\rangle\left\langle\langle u, y\rangle-f_{t}(y)\right.$. On the other hand, int $F$ is contained in the set of the Slater points of $\sigma$ (which is not empty, according to Corollary 2), by (2.7).

(iv) Let $E=F \cap\left\{x \in \mathbb{R}^{n} \mid\langle a, x\rangle=b\right\}$ and suppose that $\langle a, x\rangle \leq b$ for all $x \in F$. By Theorem $5.9(\mathrm{v})$ in $[\mathbf{3}], a \in A(y)$, for all $y \in E$, and

$$
E=\bigcap_{i \in I} F_{\left(t_{i}, y_{i}, u_{i}\right)}
$$

with $u_{i} \neq 0_{n}$, for all $i \in I$, and $I$ finite. Each $F_{\left(t_{i}, y_{i}, u_{i}\right)}$ is contained in $F_{t_{i}}$, where $f_{t_{i}}$ is not a constant function (otherwise $\partial f_{t_{i}}(x)=0_{n}$, for all $x$ ), and then $t_{i}$ is a proper index, for every $i \in I$.

Corollary 5. Let $\sigma=\left\{f_{t}(x) \leq 0, t \in T\right\}$ be a LFM convex system. Then:

(i) $\operatorname{dim} F=n$ if and only if $A(x)$ is pointed at every $x \in F$, and this happens if

$$
0_{n+1} \notin \operatorname{conv}\left(\bigcup\left\{g p h f_{t}^{*}, t \in T\right\} \backslash\left\{0_{n+1}\right\}\right) .
$$

(ii) $\operatorname{dim} F=n-\operatorname{dim} \operatorname{span}\left\{\cup_{t \in T_{C}} \cap_{y \in F} \partial f_{t}(y)\right\}$.

Proof. (i) The result is a straightforward consequence of Theorem 5.9 (ii) in $[3]$, since $A_{L}(x)=A(x)$, for all $x \in F$.

Concerning the second statement, again from Theorem 5.9 (ii) in $[\mathbf{3}], A(x)$ is pointed at every $x \in F$ if and only if

$$
0_{n+1} \notin \operatorname{conv}\left\{\left(\begin{array}{c}
u \\
\langle u, y\rangle-f_{t}(y)
\end{array}\right) \neq 0_{n+1},(t, y, u) \in T_{L}\right\} .
$$

By Theorem 23.5 in $[\mathbf{5}], u \in \partial f_{t}(y)$ is equivalent to $f_{t}(y)+f_{t}^{*}(u)=\langle u, y\rangle$, hence (4.6) can be rewritten

$$
0_{n+1} \notin \operatorname{conv}\left\{\left(\begin{array}{c}
u \\
f_{t}^{*}(u)
\end{array}\right) \neq 0_{n+1}, u \in \operatorname{range} \partial f_{t}, t \in T\right\},
$$

where

$$
\text { range } \partial f_{t}:=\bigcup\left\{\partial f_{t}(x) \mid x \in \mathbb{R}^{n}\right\} \text {, }
$$


and from the inclusion range $\partial f_{t} \subset \operatorname{dom} f_{t}^{*}([\mathbf{5}, \mathrm{p} .227])$, we get the sufficient condition (4.5).

(ii) It is a direct consequence of (4.1).

REMARK 4. If $\sigma$ is not LFM, the three subspaces which appear in (4.1) always verify the inclusions:

$$
\begin{aligned}
\operatorname{span}\left\{\cup_{t \in T_{C}} \cap_{y \in F} \partial f_{t}(y)\right\} & \subset(F-\bar{x})^{\perp}, \\
\operatorname{lin} A(\bar{x}) & \subset(F-\bar{x})^{\perp} .
\end{aligned}
$$

But these inclusions can be strict, and the subspaces $\operatorname{span}\left\{\cup_{t \in T_{C}} \cap_{y \in F} \partial f_{t}(y)\right\}$ and lin $A(\bar{x})$ do not coincide in general, as the following example shows.

Example 6. Let us consider the linear consistent system, defined in $\mathbb{R}^{2}$,

$$
\left.\left.\sigma=\left\{x_{1} \leq 0,-x_{1} \leq t, x_{2} \leq t,-x_{2} \leq t, t \in\right] 0,1\right]\right\} .
$$

Then $F=\left\{0_{2}\right\}$, but $\sigma$ is not LFM in $F$, since $D\left(F, 0_{2}\right)^{\circ}=\mathbb{R}^{2}$ and $A\left(0_{2}\right)=$ $\left\{\left(\begin{array}{l}\lambda \\ 0\end{array}\right), \lambda \geq 0\right\}$. In this case, $\left(F-0_{2}\right)^{\perp}=\mathbb{R}^{2}$ and $\operatorname{lin} A\left(0_{2}\right)=\left\{0_{2}\right\}$, whereas $\operatorname{span}\left\{a_{t}, t \in T_{C}\right\}=\left\{\left(\begin{array}{l}\lambda \\ 0\end{array}\right), \lambda \in \mathbb{R}\right\}$

Remark 5. Applying Theorem 5.9 (iv) in [3] to $\sigma_{L}$, it can be stated that int $F$ is the set of the Slater points of the (equivalent) system obtained by the elimination of the trivial inequalities in $\sigma_{L}$. This is not true, in general, for the convex system $\sigma$.

Example 7. Let us consider again the system in Example 2. We get $F=$ $[-1,1]=F_{t}$, for all $t \in T$; then $T_{C}=T$. The system $\sigma$ is LFM and the set of Slater points is empty, but int $F \neq \emptyset$.

Remark 6. Again by Theorem 5.9 (iv) in [3], when it is applied to $\sigma_{L}$, it holds that rint $F$ is the solution set of the system

$$
\left\{\begin{array}{l}
\langle u, x\rangle<\langle u, y\rangle-f_{t}(y), \quad(t, y, u) \in T_{L} \backslash T_{L, C}, \\
\langle u, x\rangle=\langle u, y\rangle-f_{t}(y), \quad(t, y, u) \in T_{L, C}
\end{array}\right\},
$$

but, in general,

$$
\operatorname{rint} F=\left\{x \in \mathbb{R}^{n} \mid f_{t}(x)<0, t \in T \backslash T_{C} ; f_{t}(x)=0, t \in T_{C}\right\},
$$

does not hold, as the following example shows.

Example 8. Let $\sigma=\left\{f_{t}(x) \leq 0, t \in T\right\}$ be a convex system, in $\mathbb{R}$, where $T=\{0\} \cup[2,3]$ and

$$
\begin{aligned}
& f_{0}(x):=\quad \begin{array}{c}
0, \text { if } x \leq 0, \\
x, \text { if } x>0 .
\end{array} \\
& f_{t}(x):=e^{x}-t, t \in[2,3] .
\end{aligned}
$$

We observe that $F=]-\infty, 0]$, the system $\sigma$ is LFM, and $T_{C}=\{0\}$. Nevertheless in this case, and since $F_{t}=\emptyset$, for all $t \in[2,3]$, 


$$
\operatorname{rint} F=\operatorname{int} F \varsubsetneqq F=\left\{x \in \mathbb{R} \mid f_{t}(x)<0, t \in[2,3] ; f_{0}(x)=0\right\} .
$$

Remark 7. Theorem 5.9 (v) in [3] shows that, in the linear case, if a supporting half-space to $F$ defines an exposed face $E$, then $E$ is the intersection of a finite number of faces $F_{t}$ of proper indices. In the convex case, we can only guarantee that $E$ is contained in the intersection of a finite number of sets $F_{t}$ of proper indices (Theorem 1 (iv)), but we cannot ensure the equality.

Example 9. Let us consider again the system in Example 8. Take $E=\{0\}$. Every index is proper, and $F_{t}=\emptyset$, for all $t \in[2,3], F_{0}=F$, and $E \varsubsetneqq F_{0}$.

\section{Characterization of the interior and the relative interior of the solution set}

As it was pointed out in Remark 5, if $\sigma=\left\{f_{t}(x) \leq 0, t \in T\right\}$ is a consistent LFM convex system, it is not guaranteed the equality

$$
\operatorname{int} F=\left\{x \in \mathbb{R}^{n} \mid f_{t}(x)<0, t \in \widetilde{T}\right\},
$$

where $\widetilde{T}=\left\{t \in T \mid f_{t}\right.$ is not identically zero $\}$; i.e, the indices set of the equivalent system $\widetilde{\sigma}$ obtained by the elimination of all the trivial inequalities in $\sigma$.

Theorem 2 will provide sufficient and necessary conditions for the fulfilment of the equality (5.1). Its proof requires the following lemma.

Lemma 1. Let $\sigma=\left\{f_{t}(x) \leq 0, t \in T\right\}$ be a consistent convex system and assume that $T_{C} \neq \emptyset$. If there exists $t \in T_{C}$ such that $\inf f_{t}<0$, then $\operatorname{int} F=\emptyset$.

Proof. Since $t \in T_{C}$, and by Proposition 5 (vi), $F=F_{(t, y, u)}$, for some $y \in F$ and $u \in \partial f_{t}(y)$; hence $F \subset\left\{x \in \mathbb{R}^{n} \mid\langle u, x\rangle=\langle u, y\rangle\right\}$. If $u=0_{n}$, then $y$ is a global minimum of $f_{t}$, but $f_{t}(y)=0$ and $\inf f_{t}<0$. We conclude that $u \neq 0_{n}$, which implies that $\operatorname{dim} F<n$ and $\operatorname{int} F=\emptyset$.

Theorem 2. Let $\sigma=\left\{f_{t}(x) \leq 0, t \in T\right\}$ be a LFM convex system and let $\widetilde{\sigma}$ be the equivalent system obtained by the elimination of the trivial inequalities. Each of the following conditions is sufficient for $\sigma$ to verify (5.1):

(i) $\widetilde{T}_{C}=\emptyset$;

(ii) $\widetilde{T}_{C} \neq \emptyset$ and there exists $\widetilde{t} \in T_{C}$ such that $\inf f_{\widetilde{t}}<0$;

(iii) $\widetilde{T}_{C} \neq \emptyset$ and there exists $\widetilde{t} \in T_{C}$ such that $f_{\widetilde{t}}$ is not differentiable at any point in $F$.

Moreover, if $\sigma$ verifies the equality (5.1), then one of the conditions (i)-(iii) must hold.

Proof. (i) If $\widetilde{T}_{C}=\emptyset$, since $F$ is the solution set of $\widetilde{\sigma}$, the result follows from Theorem 1 (iii).

We proceed now in the following way: assuming that $\widetilde{T}_{C} \neq \emptyset$, then $T_{C} \neq \emptyset$ and for all $t \in T_{C}, f_{t}$ is not identically zero. Moreover the set of Slater points of $\widetilde{\sigma}$ is empty. We shall analyze all the possibilities, and we shall see that only in the cases specified in (ii) and (iii), it happens that int $F=\emptyset$. Therefore we shall have shown the direct and the converse statements.

Case 1 (which corresponds with (ii)).

If there exists $\widetilde{t} \in T_{C}$ such that $\inf f_{\widetilde{t}}<0$, then $\operatorname{int} F=\emptyset$, by Lemma 1 .

Case 2. 
For every $t \in T_{C}$, inf $f_{t}=0$. This is equivalent to $0_{n} \in \partial f_{t}(z)$, for all $z \in F$, and every $t \in T_{C}$, then $0_{n} \in \cap_{z \in F} \partial f_{t}(z)$, for all $t \in T_{C}$.

By Corollary 5 (ii),

$$
\operatorname{dim} F=n-\operatorname{dim} \operatorname{span}\left\{\cup_{t \in T_{C}} \cap_{z \in F} \partial f_{t}(z)\right\},
$$

and two possibilities may occur:

Case 2.1.

For every $t \in T_{C}, f_{t}$ is differentiable at some point $z_{t} \in F$, then $\left\{0_{n}\right\}=\partial f_{t}\left(z_{t}\right)$, which implies that $\cup_{t \in T_{C}} \cap_{z \in F} \partial f_{t}(z)=\left\{0_{n}\right\}$. Hence, $\operatorname{dim} F=n$ and int $F \neq \emptyset$. In this case, the set of Slater points of $\widetilde{\sigma}$ does not agree with int $F$.

Case 2.2 (which corresponds with (iii)).

There exists $\tilde{t} \in T_{C}$ such that $f_{\widetilde{t}}$ is not differentiable at any point in $F$. Then, by Theorem 25.5 in [5], the set of points $D \subset \mathbb{R}^{n}$ where $f_{\widetilde{t}}$ is differentiable is a dense subset of $\mathbb{R}^{n}$, and we have to conclude that int $F=\emptyset$ (otherwise $D \cap \operatorname{int} F \neq \emptyset$ ).

Next, we shall characterize the relative interior of the solution set $F$. The objective is, again, to provide sufficient and necessary conditions for the equality

$$
\operatorname{rint} F=\left\{x \in \mathbb{R}^{n} \mid f_{t}(x)<0, t \in T \backslash T_{C} ; f_{t}(x)=0, t \in T_{C}\right\},
$$

which is valid in the linear case, but not, in general, in the convex one (remember Remark 6).

We shall assume that $F$ is not a singleton. Otherwise, $F=\{\bar{x}\}$, and then rint $F=F$, and $f_{t}(\bar{x})=0$ if and only if $t \in T_{C}$, while $f_{t}(\bar{x})<0$ if and only if $t \in T \backslash T_{C}$, in which case, $F_{t}=\emptyset$. Hence the equality (5.2) trivially holds.

We start with a couple of necessary conditions (not involving the LFM property for $\sigma$ ), being the second one also sufficient when $\operatorname{dim} F=n$.

Proposition 6. Let $\sigma=\left\{f_{t}(x) \leq 0, t \in T\right\}$ be a consistent convex system. If the equality (5.2) holds, then there exists $t \in T \backslash T_{C}$ such that $F_{t} \neq \emptyset$.

Proof. Suppose that, for all $t \in T \backslash T_{C}, F_{t}=\emptyset$. Then for every $x \in \operatorname{rbd} F$, $f_{t}(x)<0$, if $t \in T \backslash T_{C}$, and $f_{t}(x)=0$, if $t \in T_{C}$. Taking (2.7) into account, we conclude that

$$
\operatorname{rint} F \varsubsetneqq\left\{x \in \mathbb{R}^{n} \mid f_{t}(x)<0, t \in T \backslash T_{C} ; f_{t}(x)=0, t \in T_{C}\right\},
$$

contradicting that (5.2) holds.

The following example shows that the necessary condition in Proposition 6 is not sufficient, even in the case that $F_{t} \neq \emptyset$, for all $t \in T \backslash T_{C}$. Moreover, in this example, $\sigma$ is LFM.

ExAmple 10. Let us consider the system, in $\mathbb{R}, \sigma=\left\{f_{t}(x) \leq 0, t \in T\right\}$, where $T=[1,+\infty[$, and

$$
\begin{aligned}
& f_{1}(x):= \begin{cases}-3 x-6, & \text { if } x \leq-2, \\
0, & \text { if }-2<x \leq 0, \\
x, & \text { if } x>0 .\end{cases} \\
& f_{t}(x):=x^{2}+(2-t) x-2 t, t>1 .
\end{aligned}
$$

Observe that $F=[-2,0], T_{C}=\{1\}, F_{t}=\{-2\}$, for all $t>1$, and 


$$
\operatorname{int} F \varsubsetneqq]-2,0]=\left\{x \in \mathbb{R} \mid f_{t}(x)<0, t>1 ; f_{1}(x)=0\right\} .
$$

Proposition 7. Let $\sigma=\left\{f_{t}(x) \leq 0, t \in T\right\}$ be a consistent convex system. If the equality (5.2) holds, then for all $\bar{x} \in \operatorname{rbd} F$ there exists $t \in T(\bar{x})$ such that $\inf f_{t}<0$. Moreover, if $\operatorname{dim} F=n$, the converse statement is also true.

Proof. It is evident from (2.7) that the equality (5.2) will hold if and only if for all $x \in \operatorname{rbd} F$,

$$
T(x) \cap\left(T \backslash T_{C}\right) \neq \emptyset .
$$

If there exists $\bar{x} \in \operatorname{rbd} F$ such that $\inf f_{t}=0$, for all $t \in T(\bar{x})$, then

$$
0=f_{t}(\bar{x}) \leq f_{t}(x) \leq 0,
$$

for every $x \in F$, and we get $T(\bar{x}) \subset T_{C}$, contradicting (5.3).

Now, for the converse statement, suppose that $\operatorname{dim} F=n$. We shall prove that (5.3) holds, for all $x \in \mathrm{bd} F$.

Take $\bar{x} \in \operatorname{bd} F$, and $t \in T(\bar{x})$ such that $\inf f_{t}<0$. The set

$$
G_{t}=\left\{x \in \mathbb{R}^{n} \mid f_{t}(x) \leq 0\right\},
$$

is a full-dimensional closed convex set in $\mathbb{R}^{n}$, containing $F$, which verifies $\operatorname{int} G_{t}=$ $\left\{x \in \mathbb{R}^{n} \mid f_{t}(x)<0\right\}$ and bd $G_{t}=\left\{x \in \mathbb{R}^{n} \mid f_{t}(x)=0\right\}$ ([5, Theorem 7.6]). Since $\operatorname{dim} F=n, F \cap \operatorname{int} G_{t} \neq \emptyset$ and there exists $x^{0} \in F$ such that $f_{t}\left(x^{0}\right)<0$. Thus $t \notin T_{C}$.

According to Proposition 7, the equality (5.2) does not hold in Example 10 because, for $\bar{x}=0 \in \operatorname{bd} F$, inf $f_{t}=0$, if $t \in T(\bar{x})=\{1\}$.

Next example will show that, in the case $\operatorname{dim} F<n$, the necessary condition stated in Proposition 7 is not sufficient, even if the system $\sigma$ is LFM.

Example 11. Let $\sigma=\left\{f_{t}\left(x_{1}, x_{2}\right) \leq 0, t \in T\right\}$ be a convex system, in $\mathbb{R}^{2}$, where $T=[0,1] \cup\{2\}$ and

$$
\begin{aligned}
f_{0}\left(x_{1}, x_{2}\right) & :=\left|x_{1}\right|+\left[x_{2}\right]_{+} \equiv\left|x_{1}\right|+\max \left\{0, x_{2}\right\}, \\
f_{t}\left(x_{1}, x_{2}\right) & \left.\left.:=x_{1}^{2}+t x_{2}-t, t \in\right] 0,1\right], \\
f_{2}\left(x_{1}, x_{2}\right) & :=x_{1} .
\end{aligned}
$$

The solution set is $F=\{0\} \times]-\infty, 0]$ and $T_{C}=\{0,2\}=T\left(0_{2}\right)$. We also have $\inf f_{2}<0$.We can see that $\left\{0_{2}\right\}=\operatorname{rbd} F$ and $T\left(0_{2}\right) \cap\left(T \backslash T_{C}\right)=\emptyset$. Hence, the equality (5.2) does not hold.

Next we prove that $\sigma$ is LFM.

Denoting by $g_{1}\left(x_{1}, x_{2}\right)=\left|x_{1}\right|$ and $g_{2}\left(x_{1}, x_{2}\right)=\left[x_{2}\right]_{+}$, we know that for $\bar{x}=0_{2}$, $T(\bar{x})=\{0,2\}$ and

$$
\begin{aligned}
& \partial f_{0}(\bar{x})=\partial g_{1}(\bar{x})+\partial g_{2}(\bar{x}), \\
& \partial g_{1}(\bar{x})=\left\{u \in \mathbb{R}^{2} \mid-1 \leq u_{1} \leq 1, u_{2}=0\right\}, \\
& \partial g_{2}(\bar{x})=\left\{u \in \mathbb{R}^{2} \mid u_{1}=0,0 \leq u_{2} \leq 1\right\} .
\end{aligned}
$$

Thus $\partial f_{0}(\bar{x})=\left\{u \in \mathbb{R}^{2} \mid-1 \leq u_{1} \leq 1,0 \leq u_{2} \leq 1\right\}$.

On the other hand, $\partial f_{2}(\bar{x})=\left\{\left(\begin{array}{l}1 \\ 0\end{array}\right)\right\}$. Then 


$$
A(\bar{x})=\text { cone }\left\{\partial f_{0}(\bar{x}) \cup \partial f_{2}(\bar{x})\right\}=\mathbb{R} \times\left[0,+\infty\left[=D(F, \bar{x})^{\circ} .\right.\right.
$$

Also, for $\bar{x}=\left(\begin{array}{c}0 \\ x_{2}\end{array}\right), x_{2}<0$, we get $T(\bar{x})=\{0,2\}$, and

$$
\partial g_{1}(\bar{x})=\left\{u \in \mathbb{R}^{2} \mid-1 \leq u_{1} \leq 1, u_{2}=0\right\}, \partial g_{2}(\bar{x})=\left\{0_{2}\right\} .
$$

obtain

Then $\partial f_{0}(\bar{x})=\left\{u \in \mathbb{R}^{2} \mid-1 \leq u_{1} \leq 1, u_{2}=0\right\}$ and $\partial f_{2}(\bar{x})=\left\{\left(\begin{array}{l}1 \\ 0\end{array}\right)\right\}$. We

$$
A(\bar{x})=\text { cone }\left\{\partial f_{0}(\bar{x}) \cup \partial f_{2}(\bar{x})\right\}=\mathbb{R} \times\{0\}=D(F, \bar{x})^{\circ} .
$$

Except in the case $\operatorname{dim} F=n$, there is no relationship between both necessary conditions stated in Propositions 6 and 7:

(C.1) There exists an index $t \in T \backslash T_{C}$ such that $F_{t} \neq \emptyset$;

(C.2) for all $\bar{x} \in \operatorname{rbd} F$, there exists $t \in T(\bar{x})$ such that inf $f_{t}<0$.

In fact, if $\operatorname{dim} F=n,($ C.2) is equivalent to the fulfilment of the equality (5.2), and it implies (C.1). But (C.1) does not imply (C.2), as Example 10 shows.

On the other hand, if $\operatorname{dim} F<n$, the system in Example 11 verifies (C.2), but for all $\left.\left.t \in T \backslash T_{C}=\right] 0,1\right], F_{t}=\emptyset$, and therefore (C.1) fails. Finally, the following example shows that (C.1) does not imply (C.2).

Example 12. Let $\sigma=\left\{f_{t}\left(x_{1}, x_{2}\right) \leq 0, t \in T\right\}$ be a convex system, in $\mathbb{R}^{2}$, where $T=[1,2[$ and

$$
\begin{aligned}
& f_{1}\left(x_{1}, x_{2}\right):=\left|x_{1}\right|+\left[x_{2}\right]_{+}, \\
& \left.f_{t}\left(x_{1}, x_{2}\right):=x_{2}^{2}+(2-t) x_{2}-2 t, t \in\right] 1,2[.
\end{aligned}
$$

We have $F=\{0\} \times[-2,0]$ and $T_{C}=\{1\}$. For all $t \in T \backslash T_{C}, F_{t}=\left\{\left(\begin{array}{c}0 \\ -2\end{array}\right)\right\}$, and (C.1) holds.

On the other hand, for $\bar{x}=0_{2} \in \operatorname{rbd} F, T(\bar{x})=\{1\}$, and inf $f_{1}=0$. Hence, (C.2) fails.

As in previous examples, this system is LFM:

For $\bar{x}=0_{2}$ (it was calculated in Example 11),

$$
\partial f_{1}(\bar{x})=\left\{u \in \mathbb{R}^{2} \mid-1 \leq u_{1} \leq 1,0 \leq u_{2} \leq 1\right\}
$$

then $A(\bar{x})=$ cone $\left\{\partial f_{1}(\bar{x})\right\}=\mathbb{R} \times\left[0,+\infty\left[=D(F, \bar{x})^{\circ}\right.\right.$.

$$
\text { If } \begin{aligned}
\bar{x}=\left(\begin{array}{c}
0 \\
-2
\end{array}\right), T(\bar{x})=T, & \text { and } \\
\partial f_{1}(\bar{x}) & =\left\{u \in \mathbb{R}^{2} \mid-1 \leq u_{1} \leq 1, u_{2}=0\right\} \\
\partial f_{t}(\bar{x}) & \left.=\left\{\nabla f_{t}(\bar{x})\right\}=\left\{\left(\begin{array}{c}
0 \\
-2-t
\end{array}\right)\right\}, t \in\right] 1,2[.
\end{aligned}
$$

(See Example 11 for the calculus of $\partial f_{1}(\bar{x})$, when $x_{2}<0$.)

Hence, $A(\bar{x})=$ cone $\left.\left.\left\{\bigcup \partial f_{t}(\bar{x}), t \in T\right\}=\mathbb{R} \times\right]-\infty, 0\right]=D(F, \bar{x})^{\circ}$.

Finally, for $\bar{x}=\left(\begin{array}{c}0 \\ x_{2}\end{array}\right),-2<x_{2}<0$, we get $T(\bar{x})=\{1\}$, and

$$
\partial f_{1}(\bar{x})=\left\{u \in \mathbb{R}^{2} \mid-1 \leq u_{1} \leq 1, u_{2}=0\right\},
$$


obtaining $A(\bar{x})=\mathbb{R} \times\{0\}=D(F, \bar{x})^{\circ}$.

We focus now on the LFM systems, in order to provide sufficient conditions for the fulfilment of the equality (5.2). First, consider the following lemmas.

Lemma 2. Let $\sigma=\left\{f_{t}(x) \leq 0, t \in T\right\}$ be a LFM convex system and $\bar{x} \in \operatorname{rbd} F$. Then, there exist $t \in T(\bar{x})$ and $u \in \partial f_{t}(\bar{x})$ such that, for all $x \in F,\langle u, x\rangle \leq\langle u, \bar{x}\rangle$, and $\langle u, z\rangle<\langle u, \bar{x}\rangle$, for some $z \in F$.

Proof. Taking account of (4.7) in Remark 6 , if $\bar{x} \in \operatorname{rbd} F$ it must be

$$
T_{L}(\bar{x}) \cap\left(T_{L} \backslash T_{L, C}\right) \neq \emptyset .
$$

On the other hand, according to $[\mathbf{3},(7.11)]$, we have

$$
T_{L}(\bar{x})=\left\{(t, \bar{x}, u) \mid t \in T(\bar{x}) \text { and } u \in \partial f_{t}(\bar{x})\right\} .
$$

Hence, there exists $t \in T(\bar{x})$ and $u \in \partial f_{t}(\bar{x})$ such that $(t, \bar{x}, u) \notin T_{L, C}$, and this implies, since $f_{t}(\bar{x})=0$, that for all $x \in F,\langle u, x\rangle \leq\langle u, \bar{x}\rangle$ and $\langle u, z\rangle\langle\langle u, \bar{x}\rangle$ for some $z \in F$.

Let $\sigma=\left\{f_{t}(x) \leq 0, t \in T\right\}$ be a LFM convex system and $\bar{x} \in \operatorname{rbd} F$. We introduce the set

$U_{\bar{x}}:=\left\{u \in \mathbb{R}^{n} \mid\langle u, x\rangle \leq\langle u, \bar{x}\rangle\right.$, for all $x \in F ;\langle u, z\rangle<\langle u, \bar{x}\rangle$, for some $\left.z \in F\right\}$.

Obviously, $U_{\bar{x}}=N(F, \bar{x}) \backslash(F-\bar{x})^{\perp}$.

Lemma 3. Let $\sigma=\left\{f_{t}(x) \leq 0, t \in T\right\}$ be a LFM convex system and $\bar{x} \in \operatorname{rbd} F$. $U_{\bar{x}}$ is a non-empty convex set, such that, if $t \in T(\bar{x})$ verifies $\partial f_{t}(\bar{x}) \cap U_{\bar{x}} \neq \emptyset$, then $\operatorname{rint} \partial f_{t}(\bar{x}) \subset U_{\bar{x}}$.

Proof. The first statement comes from Lemma 2.

Now, let $t \in T(\bar{x})$ with $\partial f_{t}(\bar{x}) \cap U_{\bar{x}} \neq \emptyset$. Take $u \in \partial f_{t}(\bar{x}) \cap U_{\bar{x}}$. If $v$ is any point in rint $\partial f_{t}(\bar{x})$ other than $u$, then, according to Theorem 6.4 in [5], there exists $\mu>1$ such that $w:=(1-\mu) u+\mu v \in \partial f_{t}(\bar{x})$. Hence $v=\lambda w+(1-\lambda) u$, with $\lambda=\frac{1}{\mu}$. Since $\langle w, x\rangle \leq\langle w, \bar{x}\rangle$, for all $x \in F$, it follows that $v \in U_{\bar{x}}$.

Theorem 3. Let $\sigma=\left\{f_{t}(x) \leq 0, t \in T\right\}$ be a LFM convex system verifying the condition (C.2). Each of the following conditions is sufficient for $\sigma$ to verify the equality (5.2):

(i) $\operatorname{dim} F=n$;

(ii) $\operatorname{dim} F<n$ and, for all $\bar{x} \in \operatorname{rbd} F$,

$$
\left(\bigcup\left\{\partial f_{t}(\operatorname{rint} F), t \in T(\bar{x})\right\}\right) \cap U_{\bar{x}} \neq \emptyset .
$$

Proof. (i) It was proved in Proposition 7.

(ii) We shall show that, for all $\bar{x} \in \operatorname{rbd} F, T(\bar{x}) \cap\left(T \backslash T_{C}\right) \neq \emptyset$.

For $\bar{x} \in \operatorname{rbd} F$, we have $U_{\bar{x}} \neq \emptyset$, by Lemma 3 . For each $u \in U_{\bar{x}}$, let us consider the half-space

$$
H_{u}=\left\{x \in \mathbb{R}^{n} \mid\langle u, x\rangle \leq\langle u, \bar{x}\rangle\right\} .
$$

We have $F \subset H_{u}$, but $F$ is not entirely contained in bd $H_{u}$. Then, by Corollary 6.5.2 in [5], $\operatorname{rint} F \subset \operatorname{int} H_{u}$. Let us observe that this condition implies that if $u \in U_{\bar{x}}$, then $\langle u, y\rangle<\langle u, \bar{x}\rangle$, for all $y \in \operatorname{rint} F$. 
Since (5.4) holds, there exist $t \in T(\bar{x})$, and $y \in \operatorname{rint} F$ such that $\partial f_{t}(y) \cap U_{\bar{x}} \neq \emptyset$. Take $v \in \partial f_{t}(y) \cap U_{\bar{x}}$. Then

$$
0=f_{t}(\bar{x}) \geq f_{t}(y)+\langle u, \bar{x}-y\rangle, \text { and }\langle u, \bar{x}-y\rangle>0 .
$$

Hence $f_{t}(y)<0$, which implies that $t \notin T_{C}$.

REMARK 8. Now, we can see what fails in the system of Example 11, where $\operatorname{dim} F<n$, and the condition (C.2) holds, but not the equality (5.2): for $\bar{x}=0_{2}$, $U_{\bar{x}}=\left\{u \in \mathbb{R}^{2} \mid u_{2}>0\right\}$, whereas $\bigcup\left\{\partial f_{t}(\operatorname{rint} F), t \in T(\bar{x})\right\} \subset \mathbb{R} \times\{0\}$.

Theorem 4. Let $\sigma=\left\{f_{t}(x) \leq 0, t \in T\right\}$ be a LFM convex system verifying that, for every $t \in T_{C}$ and for all $\bar{x} \in \operatorname{rbd} F$,

$$
\partial f_{t}(\bar{x}) \subset(F-\bar{x})^{\perp} .
$$

Then the equality (5.2) holds, as well as the following statements:

(i) For all $\bar{x} \in F$

$$
\operatorname{lin} A(\bar{x})=(F-\bar{x})^{\perp}=\operatorname{span}\left\{\cup_{t \in T_{C}} \cap_{y \in \operatorname{rbd} F} \partial f_{t}(y)\right\} .
$$

(ii) $\operatorname{dim} F=n-\operatorname{dim} \operatorname{span}\left\{\cup_{t \in T_{C}} \cap_{y \in \operatorname{rbd} F} \partial f_{t}(y)\right\}$.

Proof. The first assertion follows again from the fact that $T(\bar{x}) \cap\left(T \backslash T_{C}\right) \neq \emptyset$ if $\bar{x} \in \operatorname{rbd} F$. Actually, by Lemma 2, there exist $t \in T(\bar{x})$ and $u \in \partial f_{t}(\bar{x})$ such that, for all $x \in F,\langle u, x\rangle \leq\langle u, \bar{x}\rangle$ and $\langle u, z\rangle\left\langle\langle u, \bar{x}\rangle\right.$ for some $z \in F$. Hence $u \notin(F-\bar{x})^{\perp}$ and, by hypothesis, $t \notin T_{C}$.

On the other hand, we know from Theorem 1 (i), that

$$
\operatorname{lin} A(\bar{x})=(F-\bar{x})^{\perp}=\operatorname{span}\left\{\cup_{t \in T_{C}} \cap_{y \in F} \partial f_{t}(y)\right\} \text {, for all } \bar{x} \in F,
$$

and

$$
\operatorname{span}\left\{\cup_{t \in T_{C}} \cap_{y \in F} \partial f_{t}(y)\right\} \subset \operatorname{span}\left\{\cup_{t \in T_{C}} \cap_{y \in \operatorname{rbd} F} \partial f_{t}(y)\right\} \text {. }
$$

Suppose now that $v \in \partial f_{t}(y)$, for all $y \in \operatorname{rbd} F$ and certain $t \in T_{C}$. Then, by hypothesis, $v \in(F-y)^{\perp}$. Hence, for all $x \in F$ and for all $z \in \mathbb{R}^{n}$, since $f_{t}(y)=f_{t}(x)=0$, we have

$$
f_{t}(z) \geq\langle v, z-y\rangle=\langle v, z-x\rangle
$$

which implies that $v \in \partial f_{t}(x)$, for all $x \in F$. Therefore we get (i), and (ii) is a direct consequence of it.

The hypothesis stated in Theorems 3 and 4 guarantee the fulfilment of the equality (5.2)

$$
\operatorname{rint} F=\left\{x \in \mathbb{R}^{n} \mid f_{t}(x)<0, t \in T \backslash T_{C} ; f_{t}(x)=0, t \in T_{C}\right\} .
$$

For the case $\operatorname{dim} F=n$, the condition in Theorem 3 is implied by the condition in Theorem 4, since the equality (5.2) is equivalent to (C.2), according to Proposition 7. The following examples show that there are no more implications. 
Example 13. Let $\sigma=\left\{f_{t}\left(x_{1}, x_{2}\right) \leq 0, t \in T\right\}$ be a convex system, in $\mathbb{R}^{2}$, where $T=[0,1] \cup\{2\}$ and

$$
\begin{aligned}
& f_{0}\left(x_{1}, x_{2}\right):=\left|x_{1}\right|+\left[x_{2}\right]_{+}, \\
& \left.\left.f_{t}\left(x_{1}, x_{2}\right):=x_{1}^{2}+t x_{2}, t \in\right] 0,1\right], \\
& f_{2}\left(x_{1}, x_{2}\right):=x_{1} .
\end{aligned}
$$

The feasible set is $F=\{0\} \times]-\infty, 0]$ and $T_{C}=\{0,2\}$. In this case, $T\left(0_{2}\right)=T$ and we have inf $f_{2}<0$. Let us check that $\sigma$ is LFM.

For $\bar{x}=0_{2}$,

$$
\partial f_{0}(\bar{x})=\left\{u \in \mathbb{R}^{2} \mid-1 \leq u_{1} \leq 1,0 \leq u_{2} \leq 1\right\}, \partial f_{2}(\bar{x})=\left\{\left(\begin{array}{l}
1 \\
0
\end{array}\right)\right\},
$$

as we have seen in Example 11.

On the other hand, $\left.\left.\partial f_{t}(\bar{x})=\left\{\left(\begin{array}{l}0 \\ t\end{array}\right)\right\}, t \in\right] 0,1\right]$. Then

$$
A(\bar{x})=\text { cone }\left\{\bigcup \partial f_{t}(\bar{x}), t \in T\right\}=\mathbb{R} \times\left[0,+\infty\left[=D(F, \bar{x})^{\circ} .\right.\right.
$$

Now, for $\bar{x}=\left(\begin{array}{c}0 \\ x_{2}\end{array}\right), x_{2}<0, A(\bar{x})=D(F, \bar{x})^{\circ}$, because we have the same active constraints at $\bar{x}$ as in Example 11.

This system verifies condition (ii) in Theorem 3 , since for $\bar{x}=0_{2}$,

$$
U_{\bar{x}}=\left\{u \in \mathbb{R}^{2} \mid u_{2}>0\right\},
$$

and taking any $t \in] 0,1]$, for all $\left(\begin{array}{c}0 \\ x_{2}\end{array}\right) \in \operatorname{rint} F, \partial f_{t}\left(0, x_{2}\right)=\left\{\left(\begin{array}{l}0 \\ t\end{array}\right)\right\} \subset U_{\bar{x}}$. But the hypothesis in Theorem 4, fails, because

$$
\partial f_{0}(\bar{x}) \nsubseteq(F-\bar{x})^{\perp}=\mathbb{R} \times\{0\} .
$$

ExAmple 14. Let $\sigma=\left\{f_{t}\left(x_{1}, x_{2}, x_{3}\right) \leq 0, t \in T\right\}$ be a convex system, in $\mathbb{R}^{3}$, where $T=[0,+\infty[$ and

$$
\begin{aligned}
& f_{0}\left(x_{1}, x_{2}, x_{3}\right):=\max \left\{\frac{\sqrt{x_{1}^{2}+x_{2}^{2}}-\left(x_{1}+x_{2}\right)}{2},-x_{1},-x_{2}\right\}, \\
& f_{t}\left(x_{1}, x_{2}, x_{3}\right):=t\left|x_{3}\right|, t>0 .
\end{aligned}
$$

The convexity of the function $f_{0}$ on $\mathbb{R}^{3}$ is a trivial consequence of the convexity of the function

$$
g\left(x_{1}, x_{2}, x_{3}\right)=\sqrt{x_{1}^{2}+x_{2}^{2}} .
$$

Actually $g$ is nothing else that the Euclidean norm restricted to the subspace $x_{3}=0$.

We can easily verify that

$$
F=\left\{x \in \mathbb{R}^{3} \mid x_{1} \geq 0, x_{2} \geq 0, x_{3}=0\right\}
$$


and, so, $\operatorname{dim} F=2$. The next step is to check that $\sigma$ is LFM and, to this aim, we analyze each $\bar{x} \in F$.

(1) For $\bar{x}=0_{3}, T(\bar{x})=T$. It can be seen that, for $t>0$,

$$
\partial f_{t}(\bar{x})=\left\{u \in \mathbb{R}^{3}\left|u_{1}=u_{2}=0,\right| u_{3} \mid \leq t\right\} .
$$

Applying the well-known Valadier formula (see, for instance, [3, Theorem A.17]), Theorem 23.8 in [5], and that

$$
\partial g(\bar{x})=\left\{u \in \mathbb{R}^{3} \mid u_{1}^{2}+u_{2}^{2} \leq 1, u_{3}=0\right\}
$$

(see, for instance, [6, Example VI.3.1]), we have

$$
\partial f_{0}(\bar{x})=\operatorname{conv}\left(\left\{\frac{1}{2}\left(\begin{array}{c}
u_{1}-1 \\
u_{2}-1 \\
0
\end{array}\right) \mid u_{1}^{2}+u_{2}^{2} \leq 1\right\} \cup\left\{\left(\begin{array}{c}
-1 \\
0 \\
0
\end{array}\right),\left(\begin{array}{c}
0 \\
-1 \\
0
\end{array}\right)\right\}\right) .
$$

From (5.5) and (5.6), we get

$$
\begin{aligned}
A(\bar{x}) & =\operatorname{cone}\left\{\partial f_{0}(\bar{x}) \cup\left(\bigcup_{t>0} \partial f_{t}(\bar{x})\right)\right\} \\
& =]-\infty, 0] \times]-\infty, 0] \times \mathbb{R} \\
& =D(F, \bar{x})^{\circ} .
\end{aligned}
$$

(2) For $\bar{x}=\left(\begin{array}{c}x_{1} \\ 0 \\ 0\end{array}\right)$, with $x_{1}>0$, again $T(\bar{x})=T$.

For $t>0,(5.5)$ holds, but now $g$ is differentiable and in this case, $\partial g(\bar{x})=$ $\{\nabla g(\bar{x})\}=\left\{\left(\begin{array}{c}0 \\ -\frac{1}{2} \\ 0\end{array}\right)\right\}$, hence

$$
\partial f_{0}(\bar{x})=\operatorname{conv}\left\{\left(\begin{array}{c}
0 \\
-\frac{1}{2} \\
0
\end{array}\right),\left(\begin{array}{c}
0 \\
-1 \\
0
\end{array}\right)\right\} .
$$

From (5.5) and (5.7) we obtain

$$
A(\bar{x})=\{0\} \times]-\infty, 0] \times \mathbb{R}=D(F, \bar{x})^{\circ} .
$$

(3) For $\bar{x}=\left(\begin{array}{c}0 \\ x_{2} \\ 0\end{array}\right), x_{2}>0$, use a symmetric reasoning to the one followed in $(2)$.

$$
\begin{gathered}
\text { (4) For } \left.\bar{x}=\left(\begin{array}{c}
x_{1} \\
x_{2} \\
0
\end{array}\right), x_{1}>0 \text { and } x_{2}>0, T(\bar{x})=\right] 0,+\infty[\text {, and (5.5) yields } \\
A(\bar{x})=\left\{0_{2}\right\} \times \mathbb{R}=D(F, \bar{x})^{\circ} .
\end{gathered}
$$

Therefore, $\sigma$ is a convex LFM system. 
The assumptions in Theorem 4 are satisfied since, for every $\left.t \in T_{C}=\right] 0,+\infty[$, and for all $\bar{x} \in \operatorname{rbd} F$, we have

$$
\partial f_{t}(\bar{x})=\left\{u \in \mathbb{R}^{3}\left|u_{1}=u_{2}=0,\right| u_{3} \mid \leq t\right\} \subset(F-\bar{x})^{\perp} .
$$

However, the assumptions in Theorem 3 are not fulfilled. Actually, if $\bar{x}=$ $\left(\begin{array}{c}x_{1} \\ 0 \\ 0\end{array}\right)$, with $x_{1}>0$, we have $T(\bar{x})=T$ and

$$
U_{\bar{x}}=\left\{u \in \mathbb{R}^{3} \mid u_{1}=0, u_{2}<0\right\} .
$$

If $x=\left(\begin{array}{c}x_{1} \\ x_{2} \\ 0\end{array}\right) \in \operatorname{rint} F$, we observe that $\partial f_{t}(x) \cap U_{\bar{x}}=\emptyset$, for all $t>0$. Moreover

$$
\partial f_{0}(x)=\left\{\nabla f_{0}(x)\right\}=\left\{\frac{1}{2}\left(x_{1}^{2}+x_{2}^{2}\right)^{-\frac{1}{2}}\left(\begin{array}{c}
x_{1} \\
x_{2} \\
0
\end{array}\right)-\frac{1}{2}\left(\begin{array}{l}
1 \\
1 \\
0
\end{array}\right)\right\} .
$$

Since $x_{2} \neq 0$, we have

$$
x_{1}\left(x_{1}^{2}+x_{2}^{2}\right)^{-\frac{1}{2}}-1<0,
$$

and we conclude that $\partial f_{0}(x) \cap U_{\bar{x}}=\emptyset$.

Example 15. Let us consider the convex system $\sigma=\left\{f_{t}(x) \leq 0, t \in T\right\}$, in $\mathbb{R}$, where $T=[1,2]$ and

$$
\begin{aligned}
& f_{1}(x):=x^{2}-1, \\
& f_{t}(x):= \begin{cases}-t x-t, & \text { if } x \leq 1 \\
0, & \text { if }-1<x<1, \quad \text { for } 1<t \leq 2 . \\
t x-t, & \text { if } x \geq 1\end{cases}
\end{aligned}
$$

The feasible set is $\left.\left.F=[-1,1], T_{C}=\right] 1,2\right]$ and $F_{1}=\{-1,1\}$. The system $\sigma$ is LFM and, for $\bar{x}_{1}=-1, \bar{x}_{2}=1$, we can take $t=1$, which verifies $\inf f_{1}<0$. Since $\operatorname{dim} F=1$, we are in case (i) of Theorem 3. But conditions of Theorem 4 are not held for this system: take $\bar{x}=-1$ and $2 \in T_{C}$. Then $-2 \in \partial f_{2}(\bar{x})$, but $(F-\bar{x})^{\perp}=\{0\}$.

\section{References}

[1] GOBERnA, M.A., and LOPEZ, M.A., Dimension and finite reduction in Linear Semi-Infinite Programming, Optimization, Vol. 25, pp.143-160, 1992.

[2] PUENTE, R. and VERA DE SERIO, V., Locally Farkas-Minkowski Linear Semi-Infinite Systems, TOP, Vol. 7, pp. 103-121, 1999.

[3] GOBERNA, M.A., and LOPEZ, M.A., Linear Semi-Infinite Optimization, John Wiley and Sons, Chichester, 1998.

[4] FAJARDO, M.D., and LOPEZ, M.A., Locally Farkas-Minkowski Systems in Convex SemiInfinite Programming, Journal of Optimization Theory and Applications, Vol. 103, No. 2, pp. 313-335, 1999.

[5] ROCKAFELlaR, R.T., Convex Analysis, Princeton University Press, Princeton, New Jersey, 1970. 
[6] HiRiart-URRUty, J.B., and LEMARECHAL, C., Convex Analysis and Minimization Algorithms, I, Springer Verlag, New York, New York, 1991.

[7] LI, W., NAHAK, C., and SINGER, I., Constraint Qualifications for Semi-Infinite Systems of Convex Inequalities, SIAM Journal Optimization, Vol. 11, No. 1, pp. 31-52, 2000. 